\title{
Investigating the Factors that Impact Patient Reported Outcomes and Experience in Elective Foot and Ankle Surgery
}

\author{
Karam Ahmad ${ }^{1 *}$ and Anand Pillai ${ }^{2}$ \\ ${ }^{1}$ University Hospital of the North Midlands, UK \\ ${ }^{2}$ University Hospital of South Manchester, UK \\ *Corresponding author: Karam Ahmad, University Hospital of the North Midlands, UK
}

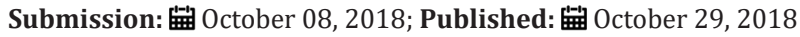

\begin{abstract}
Background: Patient reported outcome measures (PROMs) and patient reported experience measures (PREMs) have been fundamental to the NHS. PROMs2.0 allows collection and analysis of outcome data. We assess PROMs and PREMs for elective foot and ankle surgery. We also look at the use of PROMs as a new patient-based audit system, and how to improve participation.
\end{abstract}

Patients and methods: Scores used to asses PROMs- EQ-5D VAS and EQ-5D Health Index, and MOXFQPROMs data collected pre/post-operatively (follow-up 6 months) PREMs data collected using Picker Patient Experience questionnaire (PPE-15) postoperatively. 97 patients responded (27.7\% participation) Non-responders contacted to assess lack of engagement.

Results: Average MOxFQ improved- Pain: 51.4-28.2. Walking/standing:53.5-30.4. Social interaction:46.1-27.3. (p<0.00001). Average EQ-5D improved. VAS: 76.0-79.7 ( $\mathrm{p}=0.033)$, Index: 0.70-0.74. ( $\mathrm{p}=0.002)$ (Comparable to knee-arthroplasty).

Patients aged 70+-greatest MOxFQ improvement $(\mathrm{p}=0.020),<50$ and $70+/$-greatest EQ-5D improvement $(\mathrm{P}=0.01)$. Males reported better outcomes in $\operatorname{MOxFQ}(\mathrm{p}=0.024)$ Left-sided procedures better than right-not significant. Non-participation- 20/30-lack time, 7/30-No Internet, 3/30- non-specified. $66.1 \%+$ PPE-15 favourable. Asking questions and sharing of information highest-scorers $(80.8 \%+80.2 \%)$. Family involvement-most potential for improvement. (47.7\%) 55-64 age group -greatest overall satisfaction, highest in 6/7 domains. ( $\mathrm{p}=0.663$ ) Equally satisfactory experience amongst men/ women ( $\mathrm{p}=0.596)$ Results similar to published data.

Conclusion: Post-op improvement. Better outcomes with increased age and male sex. Overall satisfactory patient reported experience. PROMs are an important outcome tool of increasing importance, as we move away from manual collection. However, it still needs optimising for us to take maximum benefit from what it offers and to increase participation. Level 3-Prospective data

Keywords: Patient reported outcomes; Foot and ankle surgery; PROMs

\section{Introduction}

Patient reported outcome measures (PROMs), and patient reported experience measures (PREMs) have been a major part of the $21^{\text {st }}$ century National Health Service (NHS) [1]. Since 2009 it has been a national requirement to collect this data for the Department of Health. This initially started for elective hip and knee procedures [2], but has now extended to any form of surgery, which can make use of this data. This is a change in direction from previous years, where the only outcomes measured were physician based and used mainly for research purposes [3]. The use of PROMs allows us to move towards a more patient-centric NHS and offers us a chance to explore new patient-based audit systems. Elective foot and ankle surgery tend to be fairly complex; the impact of it on mobility and subsequently quality of life can be huge. Patient responses appear to be less predictable than hip and knee procedures. Looking at patient reported outcomes for foot and ankle surgery opens up the potential to see which factors increase the probability of better outcomes.

\section{Aims}

The aim of this study is to look at prospective PROMs and PREMs data. To investigate the relationship between patient age, gender and side of surgery on PROMs and general trends in foot and ankle surgery. Secondly, we intend to assess patient satisfaction after elective foot and ankle surgery using our PREMs data. We also investigate how to make the most of these new patient-based outcome systems, and why they are so important today.

\section{Patient and Methods}

This is a prospective study looking at all patients who underwent foot and ankle surgery. Patients reported their outcomes using a semi-automated email-based system- Amplitude. Patient outcomes were measured using various scoring systems. Firstly, the Manchester- Oxford foot questionnaire, which is a 16item patient reported outcome-measuring tool. This score has been officially validated for use in foot and ankle surgery [4]. 
Secondly, we used the 'Euroqol research foundation' derivedEQ-5D index and EQ-5D Visual analogue scale (VAS). EQ-5D-index is a generic 5-item health status measuring tool. Studies have shown the Index score to be suitable for our work, being "sensitive to changes in health status at six months' [5]. EQ-5D VAS allows a patient to rate their health on a scale from 1-100.100 being the best they can imagine, 0 being the worst. The overall EQ-5D score allows comparison of health in general, and when used together allow us to assess the change of perceived patient health post-surgery of the foot [6] (Figure 1-3).

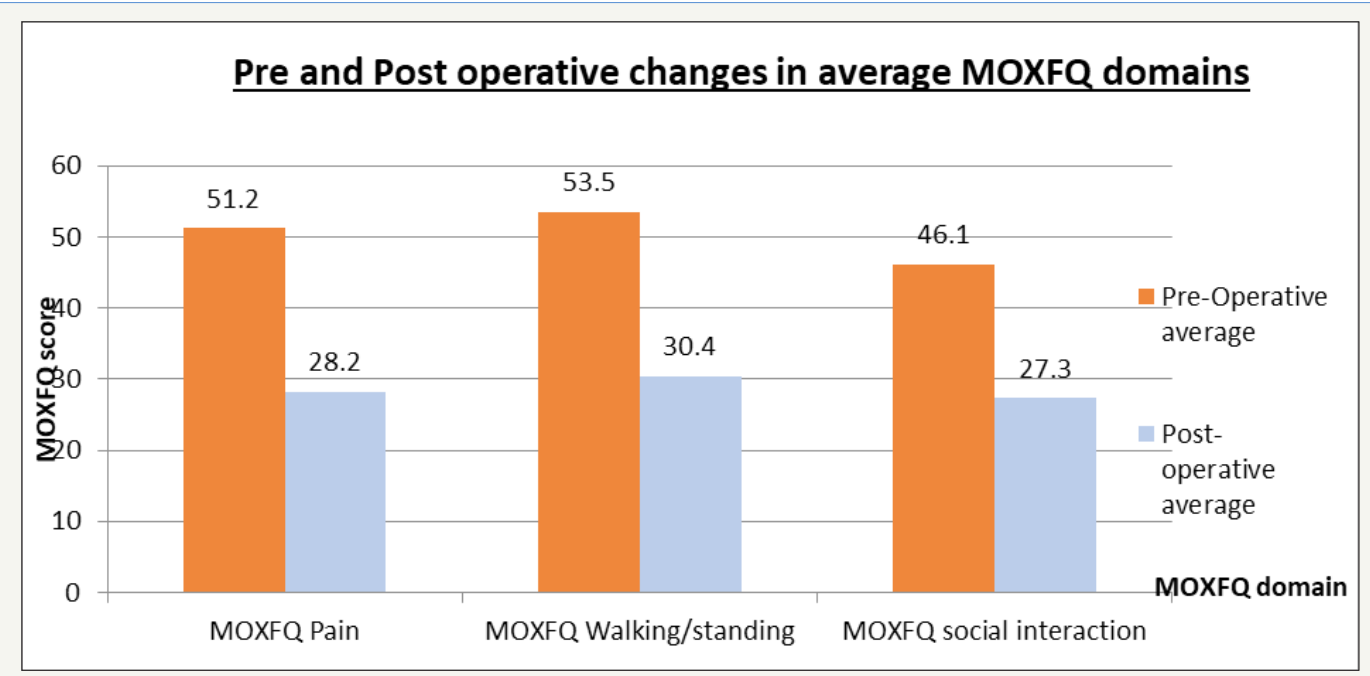

Figure 1: Pre and post-operative changes in average MOXFQ domains.

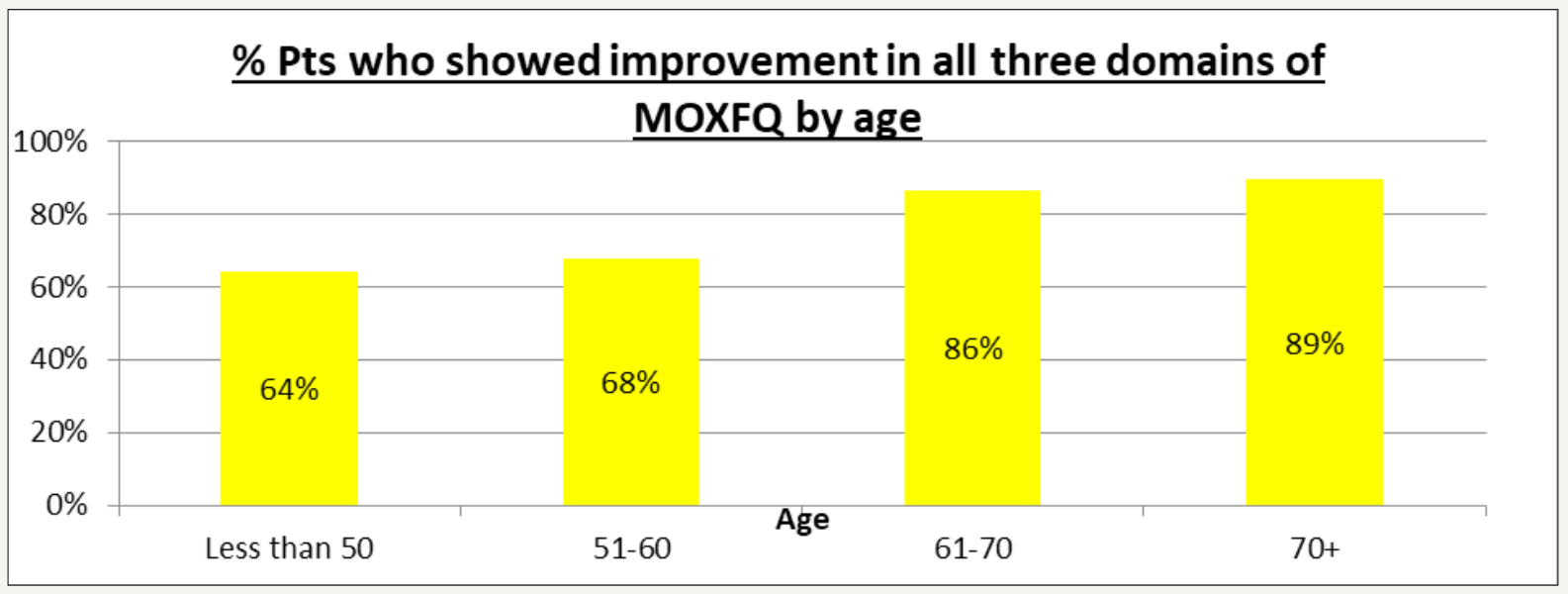

Figure 2: \% Pts who showed improvement in all three domains of MOXFQ by age.

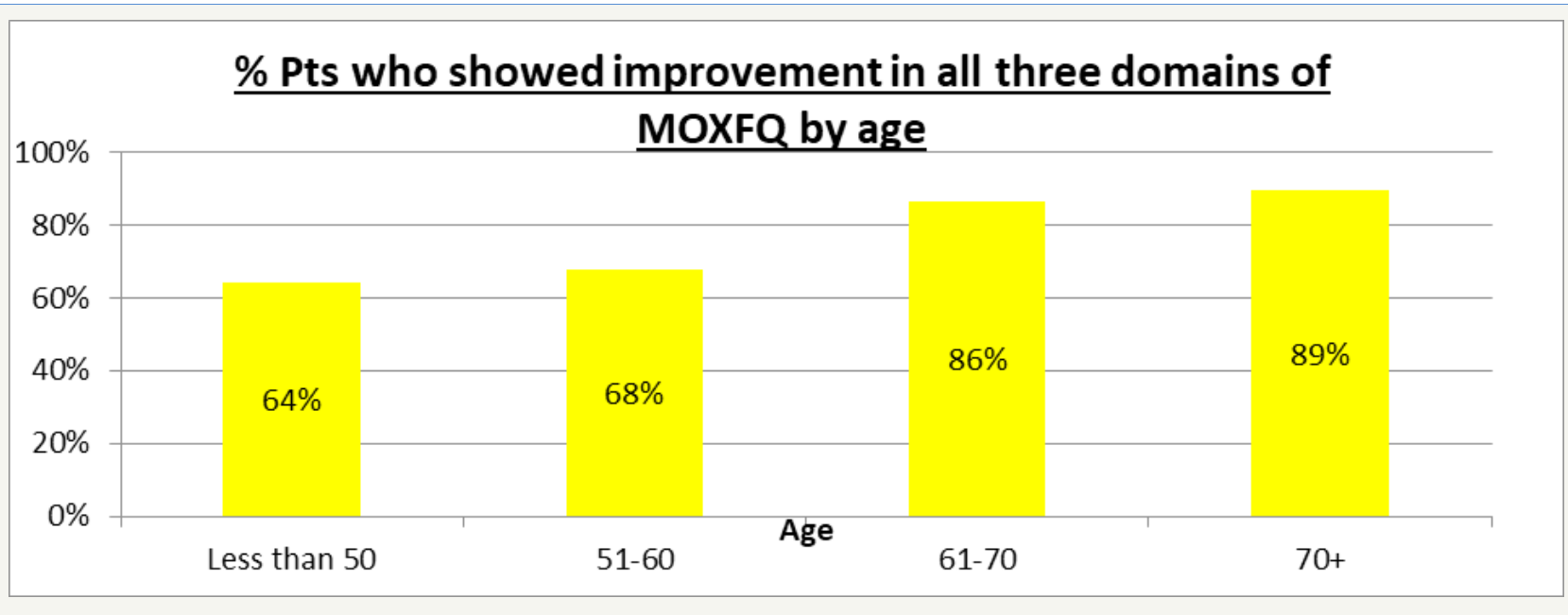

Figure 3: \% patients who improved in EQ5D VAS by age. 
Picker patient experience data (PPE-15) was collected postoperatively. Patients rated their overall experience of being in hospital, ranging from how queries were answered, passing of information, involvement in decision-making, fears, pain, involvement in care and explanation of medicines. This data was collected and overall positive responses per individual domain were summed up to give a picture of how satisfactory experience was in that particular area. All procedures were part of a single unit series and day case. All procedures were under general anesthesia and prophylactic broad-spectrum antibiotics according to local policy were given. Rehab was standardized throughout. Weight bearing was allowed post operatively, and all patients were seen at 2 weeks post op for a wound check. At six weeks physiotherapy was commenced for patients, and weight bearing AP, lateral and oblique x-rays were ordered.

Statistical tests carried out were done using the program IBM SPSS Statistics, IBM, New York. We assume a $\mathrm{p}$ value to be significant when $\mathrm{p}<0.05$. Use of T-tests ANOVA has been done where appropriate. On collection, we stratified according to diagnosis, demographics and procedure, using theatre list records. Patients who had provided preoperative PROMs scores, but not postoperative were followed up by telephone to assess their outcomes.

\section{Results}

PROMs-350 elective foot and ankle patients consented to take part in the PROMs2.0 scheme. Of this 97 (27.1\%) patients provided both pre-operative and post-operative scores. Of which 30 were followed up via calling. Of the 97 patients, 69 were female and 28 males. The average age of patients was 57.2 years with a range from $19 y$ rs to $89 y$ rs. 61 procedures were right-sided and 36 left sided. 163 patients took part in the PREMs data collection, leading to 172 pathways. Of these 50 were male and 113 females. Ages were grouped; there were 28 patients aged less than 40, 55 who were between 40-54, 49 patients between 55-64 and 40 patients above 65 . Of the 172 procedures, 96 were right sided, 73 left sided and 3 were bilateral.

\section{Patient reported outcomes}

Overall, MOxFQ scores all improved. Pain scored on average 51.4 (range 5-100) pre-operatively and improved to 28.2 (range 0-100). Walking/standing outcomes improved from pre-operatively being 53.5 (range $0-100$ ) to 30.4 (range 0-100). Social interaction also improved from pre-operatively being 46.1 (range $0-100$ ) to 27.3 (Range 0-100). ( $\mathrm{p}<0.00001)$ (Table 1 \& Figure 1).

Table 1: Average changes in MOxFQ and EQ-5D pre-operatively and post operatively in foot and ankle surgery.

\begin{tabular}{|c|c|c|c|c|}
\hline & & Pre-Op score & Post-op Score & $p$ value \\
\hline \multirow{3}{*}{ MOXFQ } & Pain-Average (range) & $51.4(5-100)$ & $28.2(0-100)$ & $<0.00001$ \\
\hline & Walking/standing Average (range) & $53.5(0-100)$ & $30.4(0-100)$ & $<0.00001$ \\
\hline & Social Interaction Average (range) & $46.1(0-100)$ & $27.3(0-100)$ & $<0.00001$ \\
\hline \multirow{2}{*}{ EQ-5D } & Index Average (range) & 0.70 (range $0.035-1$ ) & 0.74 (range $0.053-1$ ) & 0.002 \\
\hline & VAS Average (range) & $76.0(14-100)$ & $79.7(20-100)$ & 0.033 \\
\hline
\end{tabular}

EQ-5D scores showed improvement; with pre-operative index average of 0.70 (range $0.035-1$ ) improving to 0.74 (range $0.053-1$ ). $(\mathrm{p}=0.002)$ The pre-operative VAS score average of 76.0 (range 14$100)$ improved to 79.7 (range 20-100). ( $\mathrm{p}=0.033)$. 73/97 (75.3\%) patients showed improvement in all three MOxFQ fields. Of the 24 who had at least one decline, 15/24 had worsened pain, 14/24 worsened walking, and 15/24 with worsening social interactions. EQ-5D scores varied slightly, out of 89 participants, 53 (59.6\%) improved in both domains. 65/89 (73.0\%) patients had an improved EQ-5D index score and 61/89 (68.5\%) patients improved in their VAS score (Table 1).

\section{Age and PROMs}

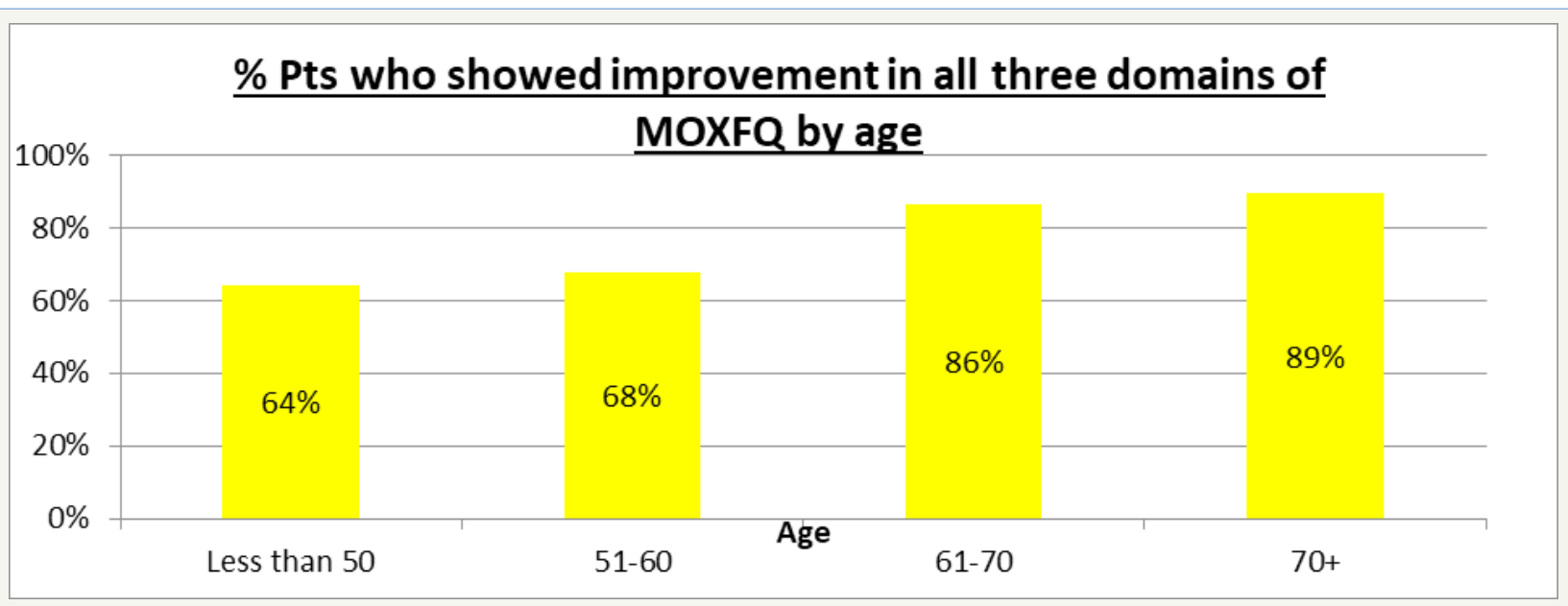

Figure 4: \% patients who improved in EQ5D Index by age. 
18/28 (64\%) patients under 50 improved in all domains, $19 / 28(68 \%)$ of those between $51-60$ years, $19 / 22(86 \%)$ of the 61-69 year group and 17/19 (89\%) of those over 70. ( $p=0.020)$ (Figure 2). EQ-5D scores followed a similar trend, for VAS scores, $19 / 25$ (76\%) patients improved under 50,13/24 (54\%) between 51-60, 13/21 (62\%) between 61-60 and 13/17 (76\%) over 70 years old. ( $p=0.011$ ). Index scores showed improvement in $18 / 25$ $(72 \%)$ of those under $50,15 / 24(63 \%)$ of those between 51-60, $16 / 21(76 \%)$ of $61-69$ and $14 / 17(82 \%)$ of those over 70 years. ( $p=$ 0.012) (Figure 3 \& 4)

\section{Gender and PROMs}

$40 / 69(58.0 \%)$ female and 23/28 (82.1\%) male patients showed improvement in all three domains of MOxFQ. ( $p=0.024)$. $36 / 89 \mathrm{EQ}-5 \mathrm{D}$ patients had some form of decline in either VAS or index. Of this 36, 28 were female, and 8 male. This gives 37/65 (56.9\%) female, and 16/24 (66.7\%) male patients who reported improvement in both VAS and index. $(\mathrm{p}=0.407)$. Individual domain scores are as follows- 17/24 (70.1\%) men had an improvement in VAS scores, and 43/66 (65.2\%) women. ( $\mathrm{p}=0.610)$. For Index scores, 19/24 (79.1\%) of men improved and 47/66 (71.2\%) women. $(\mathrm{p}=0.453)$ (Figure 5)

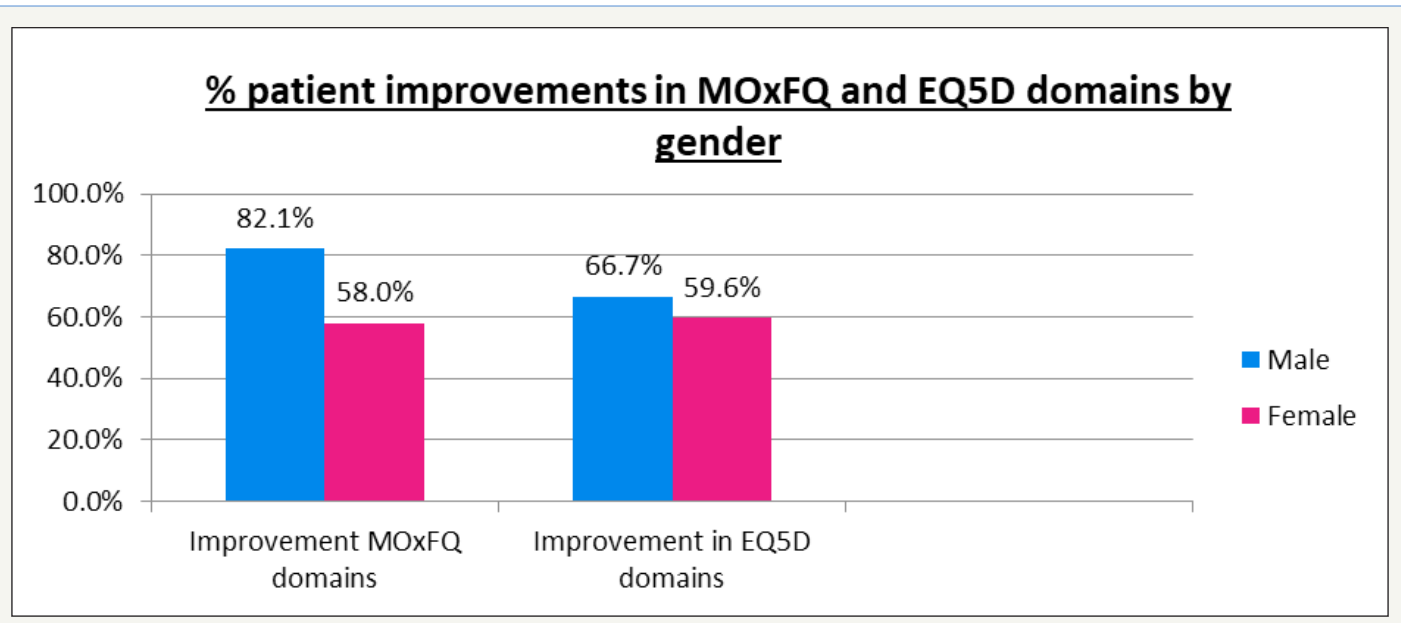

Figure 5: \% patient improvements in MOxFQ and EQ5D domains by gender.

\section{Side of surgery and PROMs}

For MOxFQ scores, 61 patients had surgery on their right side, and 36 patients had it on their left side. 44/61 (72.1\%) of patients who had a right-sided procedure reported an improvement in all three domains. $29 / 36$ patients (80.1\%) had the same result for leftsided procedures. $(\mathrm{p}=0.176)$. For EQ-5D, 35 patients underwent left sided procedures, and 54 right-sided. 24/35 (68.6\%) patients improved in both EQ-5D domains for the left side. 29/54 (53.7\%) patients who had a right-sided procedure also reported an improvement in both domains. $(\mathrm{p}=0.081)$. In terms of VAS scores, $34 / 54(63.0 \%)$ patients improved on the right side, and $26 / 35$ (74.3\%) on the left. ( $p=0.213)$. For index scores, 37/54 (69.0\%) right-sided patients improved and 28/35 (80\%) left sided patients also improved. ( $\mathrm{p}=0.134)$ (Figure 6).

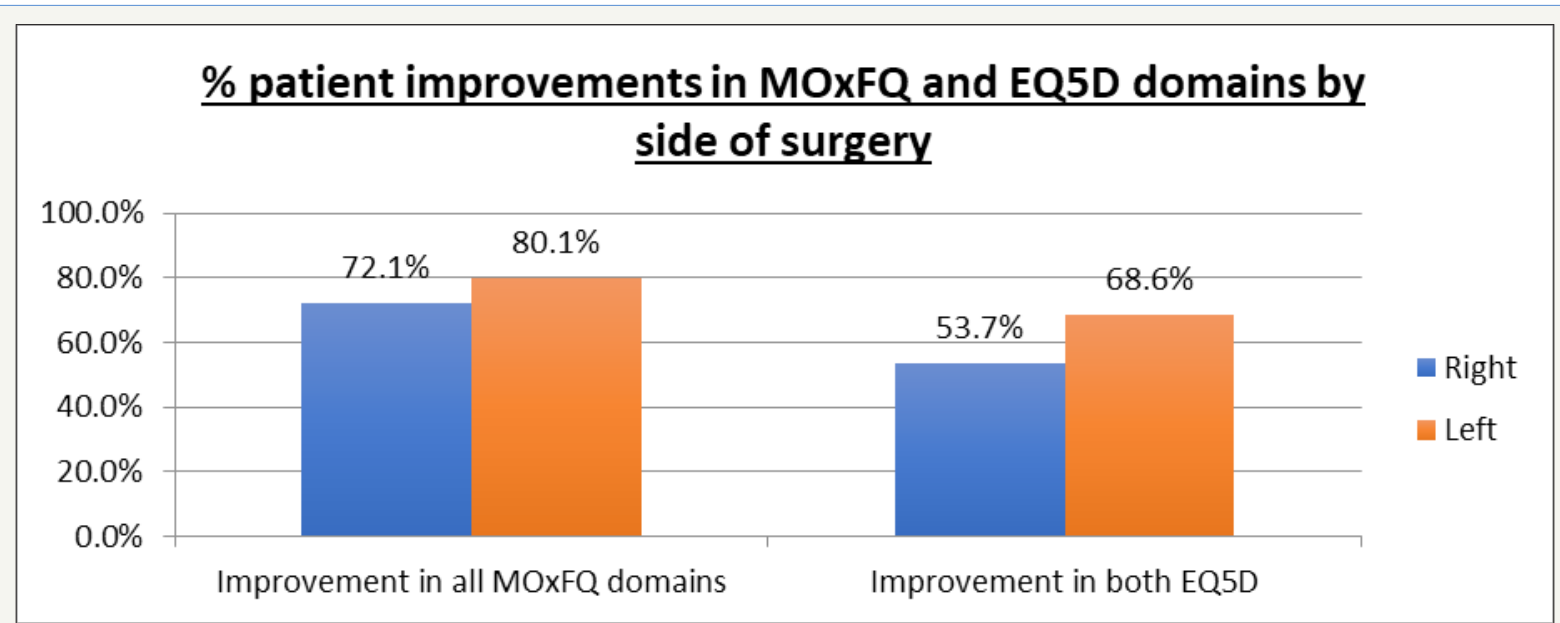

Figure 6: \% patient improvements in $\mathrm{MOxFQ}$ and $\mathrm{EQ} 5 \mathrm{D}$ domains by side of surgery.

\section{Questionnaires and participation}

There was no objective way to view differences in questionnaire, however, on asking patient who were followed up via telephone, on what questionnaire was a) easier to fill b) Most comprehensive, the over-whelming response of 25/30 (83.3\%) patients was that EQ$5 \mathrm{D}$ was easier to complete, but MOxFQ was more comprehensive in 28/30 (93.3\%). 
Reasons for non-participation in 30 patients were the following

1. 20/30 Lack of time/forgot

2. $7 / 30$ Reduced internet access

3. $3 / 30$ Did not specify a reason

\section{Patient reported experience measures}

Our patient experience data reported high overall percentage satisfaction in most domains assessed. 139/172 (80.8\%) patients reported satisfaction in being able to ask questions, 138/172

Table 2: PPE satisfaction domain variations with age.
$(80.2 \%)$ were satisfied with the way information was shared, $113 / 172(65.7 \%)$ felt they had satisfactory involvement in their care. 112/172 (65.3\%) were satisfied with discharge advice, $111 / 172(64.5 \%)$ with addressing of anxiety/fears, and 101/172 $(58.7 \%)$ with pain and its management. However just 82/172 $(47.7 \%)$ reported satisfaction when it came to family involvement. Average overall satisfaction was $66.1 \%$. Satisfaction for each domain against different age groups is shown in Table 2- $\mathrm{p}=0.663$. Satisfaction for each domain across gender is shown below in Table $3-(\mathrm{p}=0.596)$.

\begin{tabular}{|c|c|c|c|c|}
\hline & \multicolumn{3}{|c|}{ Raw and \% Average Satisfactory Response by Age Group } \\
\hline PPE-15 Domain & Less than $40(28)$ & $40-54(55)$ & $55-64(49)$ & $65+(40)$ \\
\hline Ability to ask questions & $16 / 28(57.1 \%)$ & $34 / 55(61.8 \%)$ & $48 / 49(98.0 \%)$ & $31 / 40(77.5 \%)$ \\
\hline Sharing of information & $21 / 28(75.0 \%)$ & $41 / 55(74.5 \%)$ & $44 / 49(89.8 \%)$ & $35 / 40(85.0 \%)$ \\
\hline Involvement in care and respect & $19 / 28(67.9 \%)$ & $35 / 55(63.7 \%)$ & $35 / 49(71.4 \%)$ & $29 / 40(72.5 \%)$ \\
\hline Addressing of anxiety and fears & $21 / 28(75.0 \%)$ & $38 / 55(69.1 \%)$ & $45 / 49(91.8 \%)$ & $29 / 40(72.5 \%)$ \\
\hline Pain and its management & $17 / 28(60.7 \%)$ & $32 / 55(58.2 \%)$ & $44 / 49(89.8 \%)$ & $22 / 40(55.0 \%)$ \\
\hline Opportunity to involve family & $18 / 28(64.3 \%)$ & $42 / 55(76.4 \%)$ & $40 / 49(81.6 \%)$ & $27 / 40(67.5 \%)$ \\
\hline Satisfactory discharge advice & $21 / 28(75.0 \%)$ & $36 / 55(65.5 \%)$ & $34 / 49(69.4 \%)$ & $23 / 40(57.5 \%)$ \\
\hline
\end{tabular}

Table 3: PPE satisfaction domain variations with gender.

\begin{tabular}{|c|c|c|}
\hline & F (113) & M (50) \\
\hline Ability to ask questions & $80 / 113(70.8 \%)$ & $37 / 50(74.0 \%)$ \\
\hline Sharing of information & $93 / 113(81.4 \%)$ & $40 / 50(80.0 \%)$ \\
\hline Involvement in care and respect & $79 / 113(69.9 \%)$ & $32 / 50(64.0 \%)$ \\
\hline Addressing of anxiety and fears & $92 / 113(81.4 \%)$ & $40 / 50(80.0 \%)$ \\
\hline Pain and its management & $61 / 113(54.0 \%)$ & $35 / 50(70.0 \%)$ \\
\hline Opportunity to involve family & $92 / 113(81.4 \%)$ & $36 / 50(72.0 \%)$ \\
\hline Satisfactory discharge advice & $70 / 113(62.0 \%)$ & $37 / 50(74.0 \%)$ \\
\hline
\end{tabular}

\section{Discussion}

PROMs have changed the way in which healthcare professions judge the standards of the care they provide. They were developed in 2009 [1,6], as part of a Department of Health (DoH) initiative. Their use becomes especially important where survival is not as relevant as is quality of life [7]. This makes the use of PROMs in foot and ankle surgery especially useful, as the changes in quality of life post-operatively are well known to be an important prognostic indicator to a satisfied patient. There have been countless studies looking at PROMs for hip and knee arthroplasty, and factors, which can influence them such as, implant brands and type of hospital [8]. This study has allowed us to look at demographic factors that impact PROMs. In the future there is considerable scope to look at how factors such as patients co-morbidities, and different conditions impact PROMs.

Our data for PROMs has shown a generic improvement in all three domains of MOxFQ scores that is statistically significant. Pain improved by 23.2 points, walking/standing by 23.1 points and social interaction by 18.8 points. EQ-5D scores also showed modest improvement, the average VAS score improved from 76.0 pre-operatively to 79.7 post operatively, EQ-5D index showed a significant average improvement from 0.70 to 0.74 . EQ-5D scores are also in line with current literature in that they show improvement albeit not always significant [9]. This data therefore suggests high PROMs in foot and ankle procedures. This correlates well with previous studies that have shown good outcomes in foot and ankle surgery. In 2014 83\% of patients were either unchanged or improved in their EQ-5D score, and over $80 \%$ of patients improved in their Manchester Oxford Foot questionnaire (MOxFQ) score [10]. Other studies have also shown an improvement in PROMs postoperatively, showing a mean improvement in the three domains of MOxFQ, pain, standing and walking and social interactions for podiatric surgery [5]. Another recent study has shown that arthritic conditions of the foot and hallux valgus- two extremely common conditions- have improvements post operatively in their PROMs [11] (Figure 3-6).

\section{Age}

We found that the best outcomes are in the elderly population. $86 \%$ of patients between $61-69$, and $89 \%$ of patients over 70 reported improvements in all three domains of MOxFQ. The trend could be a result of reduced expectations from the outcome of the procedure. The best EQ-5D outcomes were reported in the over $70 \mathrm{~s}$ with $76 \%$ of patients improving in their VAS score, and in the under 50 s of which $76 \%$ improved. Index scores showed the greatest $(82 \%)$ improvement in the over 70 s, followed closely at $72 \%$ by the under 50s again.

Reasons for the under 50 category becoming more prominent may be linked to the EQ-5D score being less specific then MOxFQ allowing one to respond with relation to ones overall health. Studies for knee arthroplasty suggests that patients younger than 55 , make the greatest improvement in their surgeries, but are also 
more likely to be dissatisfied [12]. When related to foot and ankle surgery, patients less than 40 placed improved walking, reduced pain and the ability to wear dress shoes as the most important outcomes when undergoing Hallux-valgus correction surgery [5]. Those between 40-60 placed pain relief as the most important, and over 60s felt improved mobility was the most important outcome. In essence we can assume that our results which suggest increasing age is a factor to better PROMs to be more reliable due to these correlations. The EQ-5D VAS is known to not be as sensitive to changes in health status post-operatively and henceforth we can still have confidence in our findings [6].

\section{Gender}

Male patients report better outcomes than females. Overall, $58.0 \%$ of female and $82.1 \%$ of male patients showed improvements in all three MOxFQ domains. EQ-5D results also followed a similar pattern, with $56.9 \%$ of females showing improvement in either VAS or index and $66.7 \%$ of men. Individual VAS and index scores showed slightly better rates of improvement in men as opposed to women. However, possibly due to the limited number of male patients, the EQ-5D results did not show statistical significance, but the trend it suggests, coupled with the above MOxFQ score means we cannot disregard it completely. The literature suggests that women place a greater importance on pain reduction, being able to wear dress shoes and overall appearance than men $[4,13]$.

\section{Side of procedure}

Left sided procedures tend to fare slightly better than right sided ones. MOxFQ scores show that $72.1 \%$ of patients reported improvement in their right-sided procedures for all three MOxFQ domains, whilst $80.1 \%$ of left sided procedures did the same. For our EQ-5D scores, again, left sided procedures fared slightly better with $68.6 \%$ improvement in both domains compared to just $53.7 \%$ on the right. The same pattern also faired for both individual VAS and Index scores. Despite these results not showing statistical significance, it would be unwise to completely disregard them, as trends are nonetheless evident.

\section{Participation}

Participation rate was just slightly over $1 / 4$ at $27.1 \%$. To improve uptake, we asked a sample of 30 patients to give their reasons for non-participation. They suggested we could improve participation by simplifying the overall process of filling out the questionnaire online, perhaps even by making patients do it on follow up appointments. Literature says that lack of knowledge or education about the program can lead to non-uptake. Patients who perceive the scheme to be of little significance also tend to not take part in it. Older patients who have perhaps more limited access to Internet struggle with participation particularly if there is an online element. Other factors that can influence low participation are being from a poor socioeconomic background, and presence of co-morbidities [14].

\section{Patient reported experience}

On evaluation of our PREMS data we found overall satisfactory experience in the majority of the domains assessed. In particular we found that the greatest satisfaction reported by patients was found to be in their ability to ask questions and the sharing of information, which were both above $80 \%$. Involvement in care, discharge advice and addressing concerns were also showing good experience outcomes with satisfaction above $60 \%$ overall. It appears slight improvement can be made when involving family, as less than $50 \%$ of patients (47.7\%) felt this was reaching their expectations.

PPE-15 studies show that patient respect, family involvement and sharing of information are the biggest factors to increase satisfaction in terms of individual domains. It summarized that communication, respect and patient engagement are the best determinants of high PREMs [15]. There is no significant trend between age and patient experience. However, some patterns have been evident in the data. The 55-64 age group shows the best experience measures in all but one of the categories. However, It has been suggested that patients who had been discharged following inpatient stay tended to show greater satisfaction with increasing age. Particularly those between 75-84 [16].

The age group with the least satisfactory experience in the majority of domains is the less than 40 category, followed by the 40-54s age group. In terms of gender, the same study as for age differences suggests there is no major difference between "patient satisfaction indexes' between men and women although the former to be more satisfied than the latter [16].

There was no statistically significant trend between gender and patient experience. With men recording the greatest satisfaction in the addressing of anxiety and fears. Women recorded their greatest satisfaction in the opportunity to involve family. The greatest difference between men and women's experience came with regards to pain and its management, where men fared better than women. The lack of statistical significance, again suggests that there is no overall difference between male and female experience. Which in itself is reassuring.

\section{Improving PROMs}

Although foot and ankle procedures are varied, one purpose of this study is to look at collection data systems and not individual outcome data on its own. Our study is an important beginning to highlighting the importance of PROMs in FAS. Our uptake rate of $27.1 \%$ may suggest that uptake in patient reported outcome surveys is low regardless of pathology.

This leads us to look at PROMs overall. We can highlight the issues, as demonstrated in our work, with newer automated and wholly electronic systems for patient outcome reporting. The strengths of PROMs are clear to see, it allows independent collection of data, away from the clinic setting, which results in less bias. It is based upon an electric system, which enables it to be environmentally friendly, allows us to quickly tabulate and graph data to recognize trends and can be done from the comfort of ones home.

The mobile nature of this scheme means its allows us to reduce the number of follow up appointments for patients. It enables us to highlight the patients who are outliers to common trends. They 
are the ones who can be seen at closer follow up intervals. This can reduce the number of clinic appointments required and therefore cost.

The weaknesses of PROMs are that it requires one to have Internet access, email access and a computer at home. More so it requires the patient to be computer literate. This may make PROMs reporting more difficult in conditions that effect elderly patient groups who may not be readily able to access the internet.

Furthermore, this style of reporting can lead to issues with take up and engagement; perhaps the direct lack of involvement of the surgical team may cause patients to perceive PROMs to lack importance. Patients may choose to ignore taking part despite email reminders; this would be much less of an issue if responses were recorded in clinics. There is also an issue of misrepresentation of outcomes. There is a risk that the patients who are on extremes of outcomes may be the ones most likely to respond. This can lead us to have results, which are either very positive or very negative with very little in between.

As with any scheme, PROMs has strengths and weaknesses. But it is clear to us on completing this study that PROMs are important. As described earlier it allows us to move towards a more patient centric health service, one in which patient reported results and satisfaction are used in tandem with radiological changes. It ensures we have a relevant and significant way to measure outcomes. In addition, it enables us to move away from expensive and labour intensive manual data collection, which cannot be supported under the current environment of financial pressures in the health service.

So, it is important to be able to find ways to increase participation. There will be many innovative ways in order to do this as we move towards a more technologically advance health service. Some of our ideas are listed below that we believe will increase participation. We believe education and engagement throughout the consultation process, including initial clinic appointments, at time of consent and pre-operatively are key to emphasize the importance of PROMs and the benefits it brings to patients. At these points, leaflets, posters and booklets can also be given.

It would be prudent to ensure those who are not confortable with computers to be able to participate. We can do this by finding viable alternatives, this may include telephone data collectionautomated or not. We could also make available appointments for data collection- this could be in hospital or in the community. In this case it will be important to maintain close links to with community teams to enable compliance to increase. This could reduce the strain on hospitals. The use of dedicated staff and calling up patients was done in parts of our study; more extensive use could allow us to assess the benefits of this.

PREMs data has had better participation; we believe this is due to patients believing the questions are more relevant to them. Therefore, we feel the use of simpler questions, reducing technical words, and making the survey more relevant to patients can improve participation. Involving patients in the changes in their condition could also increase uptake.

\section{Conclusion}

Patient reported outcomes improve after foot and ankle surgery. Older and male patients have better outcomes compared to younger and female patients. There was no significant difference between sides of surgery. Ways to improve participation will include simplification of PROMs, ensuring it is easily accessible for all patients and emphasis on the importance of data collection on all levels of healthcare. Patient reported experience data showed overall satisfactory scores in the majority of domains assessed. The greatest improvement can be made with regards to involvement of family. There was no significant trend between overall experience and age/gender.

\section{References}

1. NHS UK (2014) Monthly patient reported outcome measures (PROMs) in England a guide to proms methodology, UK.

2. Judge A, Arden NK, Kiran A (2012) Interpretation of patient-reported outcomes for hip and knee replacement surgery: identification of thresholds associated with satisfaction with surgery. J Bone Joint Surg Br 94(3): 412-418.

3. Schrier JC, Palmen LN, Verheyen CC, Jansen J, Koëter S (2015) Patientreported outcome measures in hallux valgus surgery. A review of literature. Foot Ankle Surg 21(1): 11-15.

4. Dawson J, Boller I, Doll H (2011) The MOXFQ patient-reported questionnaire: assessment of data quality, reliability and validity in relation to foot and ankle surgery. Foot (Edinb) 21(2): 92-102.

5. Maher AJ, Kilmartin TE (2012) An analysis of Euroqol EQ-5D and Manchester oxford foot questionnaire scores six months following podiatric surgery. J Foot Ankle Res 5(1): 17.

6. Dawson J, Coffey J, Doll H, Lavis G, Cooke P, et al. (2006) A patien-tbased questionnaire to assess outcomes of foot surgery: validation in the context of surgery for hallux valgus. Qual Life Res 15 (7): 1211-1222.

7. Deshpande PR, Rajan S, Sudeepthi BL, Abdul CP (2011) Patient-reported outcomes: A new era in clinical research. Perspect Clin Res 2(4): 137144

8. Baker PN, Deehan DJ, Lees D (2012) The effect of surgical factors on early patient-reported outcome measures (PROMS) following total knee replacement. J Bone Joint Surg Br 94(8): 1058-1066.

9. Jansson KÅ, Granath F (2011) Health-related quality of life (EQ-5D) before and after orthopedic surgery. Acta Orthop 82(1): 82-89.

10. Bhosale A, Chirputkar K, Pillai A (2014) PROMs 2.0 in elective foot and ankle surgery: early experience from University Hospital of South Manchester. Bone Joint J 96-B: 7.

11. Barr L, Loizou C, Smith G, Loveday D (2015) Patient-reported outcome measures for common foot and ankle conditions: the effect of disease and the benefit of surgery. Bone Joint J 97-B.

12. Williams DP, Price AJ, Beard DJ (2013) The effects of age on patientreported outcome measures in total knee replacements. Bone Joint J $95-$ $\mathrm{B}(1): 38-44$.

13. Tai CC, Ridgeway S, Ramachandran M, Ng VA, Devic N, et al. (2008) Patient expectations for hallux valgus surgery. J Orthop Surg (Hong Kong) 16(1): 91-95

14. Longtin Y, Sax H, Leape LL, Sheridan SE, Donaldson L, et al. (2010) Patient participation: current knowledge and applicability to patient safety. Mayo Clin Proc 85(1): 53-62.

15. Wong EL, Leung MC, Cheung AW, Yam CH, Yeoh EK, et al. (2011) A population-based survey using PPE-15: relationship of care aspects to patient satisfaction in Hong Kong. Int J Qual Health Care. 23(4): 390-396. 
16. Baker PN, Van der meulen JH, Lewsey J, Gregg PJ (2007) The role of pain and function in determining patient satisfaction after total knee replacement. Data from the national joint registry for england and wales. J Bone Joint Surg Br 89(7): 893-900.

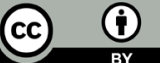

Creative Commons Attribution 4.0 International License

For possible submissions Click Here

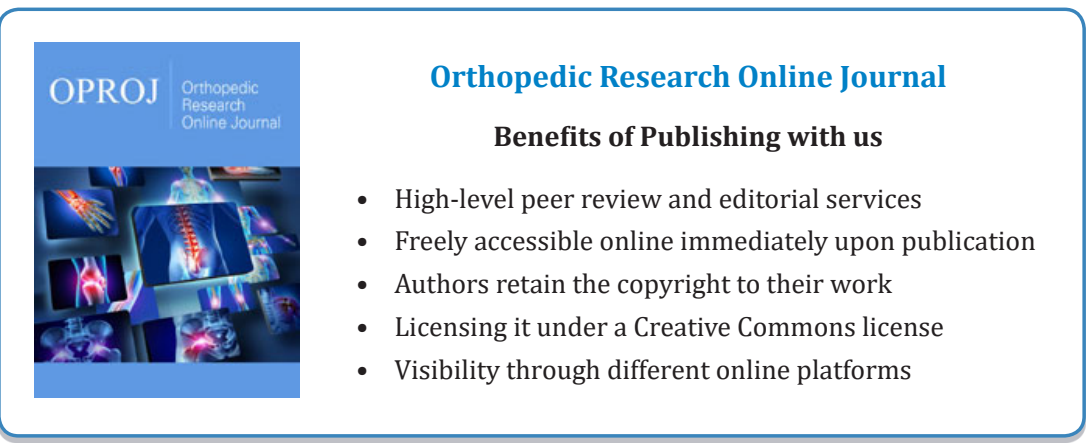

